Research Article

\title{
Use of $\mathrm{MgCl}_{2}$ Nanoparticles as Alternative Contrast Media in Magnatic Resonance Imaging Molecular Imaging and Analyzed by Voltammetric Technique
}

\author{
Dunya Ali Mustafa, Haydar Abdulkadeer Taheer Al-Shimmari, Muhammed Mizher Radhi \\ Radiological Techniques Department, Health and Medical Technology College-Baghdad, Middle Technical University (MTU), Iraq. \\ Corresponding author. E-mail: wisamhh92@gmail.com
}

Received: Mar. 19, 2020; Accepted: Apr. 13, 2020; Published: Apr. 17, 2020

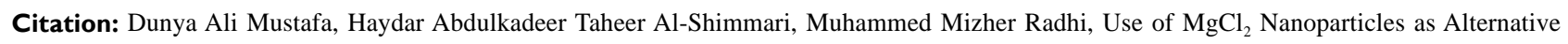
Contrast Media in Magnatic Resonance Imaging Molecular Imaging and Analyzed by Voltammetric Technique. Nano Biomed. Eng., 2020, I2(2): I48152.

DOI: 10.5101/nbe.v12i2.p148-152.

\begin{abstract}
Magnesium chloride compound nanoparticles $\left(\mathrm{MgCl}_{2} \mathrm{NPs}\right)$ were chosen as alternative contrast medium in magnetic resonance imaging (MRI). The solution of $\mathrm{MgCl}_{2} \mathrm{NPs}$ was a good electrochemical property especially in the blood medium. It was found that $\mathrm{MgCl}_{2} \mathrm{NPs}$ in blood medium served as an antioxidant reagent because cyclic voltammogram had only one reduction current peak at $0.9 \mathrm{~V}$. Gadolinium compounds were used as a common contrast medium in MRI technique; they had two oxidation current peaks in the blood medium at $0.73 \mathrm{~V}$ and $0.23 \mathrm{~V}$. In addition, the gadolinium compounds caused many problems when used as a contrast medium, but they were the only contrast media used for this purpose. It was found that using $\mathrm{MgCl}_{2} \mathrm{NPs}_{\text {solution }}$ as an alternative contrast medium in rabbits enhanced MR imaging of kidney organ, as compared with that using gadolinium at the same dose.
\end{abstract}

Keywords: $\mathrm{MgCl}_{2}$ NPs; Contrast medium of MRI; Redox; Cyclic voltammetry; Rabbit blood sample

\section{Introduction}

Contrast refers to signal difference between adjacent regions, including those between tissue and tissue, between tissue and vessel, and between tissue and bone [1]. Contrast media (CM) improves imaging quality by improving the vision of specific organs, blood vessels and tissues, which is invaluable for accurate diagnosis. Gadolinium based CM, which is used for magnetic resonance imaging (MRI), was approved for parenteral use in the late 1980s [2].

It was used as contrast agents in MRI for early diagnosis of various diseases. Due to its effect on the proton relaxation process, it modulates the intensity of the MRI signal, thus improving informative value of images and making them more contrast [3, 4].

Due to their large magnetic moment, one of the most important applications of iron oxide nanoparticles is to be used as contrast agents in MR imaging of tumors. It has the capability to distort the local magnetic characteristics of the tissue in order to yield an enhancement in image contrast [5]. Iron oxide nanoparticles are sufficiently small so that their magnetizations can randomly flip in direction at room field. However, they have much larger magnetic susceptibility than paramagnets; thus once the external 
magnetic field disappears, the magnetism of iron oxide nanoparticles will reduce to zero immediately [6, 7].

Magnesium (Mg) is one of the 5 most common minerals in the human body and is found in more than 300 human enzymes. Mg plays a major role in the normal functioning of muscles, carbohydrate metabolism, and skeletal structure. Mg supplementation is safer for mother and infant during pregnancy [8].

$\mathrm{MgCl}_{2}$ can give high resolution to MR images. It can be used as T1 contrast medium which is a substitute for gadolinium compound, simultaneously, due to the safety and high resolution of MR imaging [9, 10].

Manganese (II) complexes have excellent potential as alternative MRI contrast agents to Gd-based probes due to their favorable relaxometric properties and good biochemical characteristics, despite the lower magnetic moment, the shorter Mn-O distance of bound water, and its generally faster exchange rate relaxation values that are quite comparable $[11,12]$.

In this study, magnesium chloride nanoparticles were used as an alternative contrast medium for each of gadolinium and magnesium chloride, which showed good result of electrochemical analysis as anti-oxidative agent and enhanced the MR imaging.

\section{Experimental Materials}

Magnevist in $0.5 \mathrm{mmol} / \mathrm{mL}$ solution for injection (gadopentetate dimeglumine) used as contrast media in MRI test was from Bayer Schering Pharma (Berlin Germany). Magnesium chloride $\left(\mathrm{MgCl}_{2}\right)$ was from Fluka (Germany). Magnesium chloride nanoparticles $\left(\mathrm{MgCl}_{2} \mathrm{NPs}\right)$ were synthesized by lyophilize method. Ascorbic acid (AA) was from Technicon Chemicals Co. (Oreq. Tournai Belgique). Blood samples of rabbits, and other chemicals and solvents were of annular grade and were used as received by the manufacturers. Double distilled water was used to prepare aqueous solutions. All solutions were deaerated oxygen by nitrogen gas for 10 - 15 min prior to making measurement.

\section{Cyclic voltammetry}

EZstat series (potentiostat / glvanostat, NuVant Systems Inc., USA), electrochemical workstations of bioanalytical system with potetiostate driven by electroanalytical measuring softwares were connected to personal computer to perform cyclic voltammogram
(CV). An Ag/AgCl (3 mol $\mathrm{KCl}$ ) and platinum wire ( $1 \mathrm{~mm}$ of diameter) were used as a reference and a counter electrode respectively. The glassy carbon electrode (GCE) was used in this study after cleaning with alumina grand and sonic technique for 10 - 15 min.

\section{Lyophylization instrument}

Lyophylization instrument from LABCONCO Company (USA) was used to prepare $\mathrm{MgCl}_{2}$ nanoparticles from microparticles by deep freezing technique.

\section{Magnetic resonance imaging (MRI) 1.5 tesla}

In this in-vivo study, magnetic resonance imaging (MRI) 1.5 tesla type (Philips, Holland) was used, and the rabbit was prepared in the apparatus of MRI.

\section{Preparation of $\mathrm{MgCl}_{2}$ nanoparticles}

Lyophylization (freeze-drying method) $\mathrm{MgCl}_{2}$ was prepared by dissolving $0.75 \mathrm{~g}$ of $\mathrm{MgCl}_{2}$ microparticles in $150 \mathrm{~mL}$ of distilled water. The suspension of $\mathrm{MgCl}_{2}$ was cooled, and the ice crystals of pure water were formed at $-18{ }^{\circ} \mathrm{C}$. The second step involved sublimation of ice from the frozen product by passing heat air from shelf of lyophylization instrument to the frozen solution in the vial. The ice sublimed and the water vapor formed passed through the dried portion of the product to the surface of the sample. The water vapor was transferred from the surface of the product through the chamber to the condenser, and the water vapor condensed on the condenser. At the end of the sublimation step, a porous plug was formed. Its pores corresponded to the spaces that were occupied by ice crystals. The third step was drying, involving the removal of absorbed water from the product. All steps must be continuous for about 48 - $72 \mathrm{~h}$ [13].

\section{Atomic force microscopy (AFM)}

Fig. 1 is the scanning atomic force microscopy (AFM) of the prepared $\mathrm{MgCl}_{2} \mathrm{NPs}$ at 31.4 - $45.7 \mathrm{~nm}$ with an average dimension of $38.8 \mathrm{~nm}$ respectively, which illustrates the morphology details of nanoparticles as spherical forms as accepted results of the conversion of magnesium chloride to nanoparticles.

\section{Results and Discussion Gadolinium compound}

Gadolinium compound was used as a contrast medium in magnetic resonance imaging (MRI), and an electrochemical analysis was performed on the 


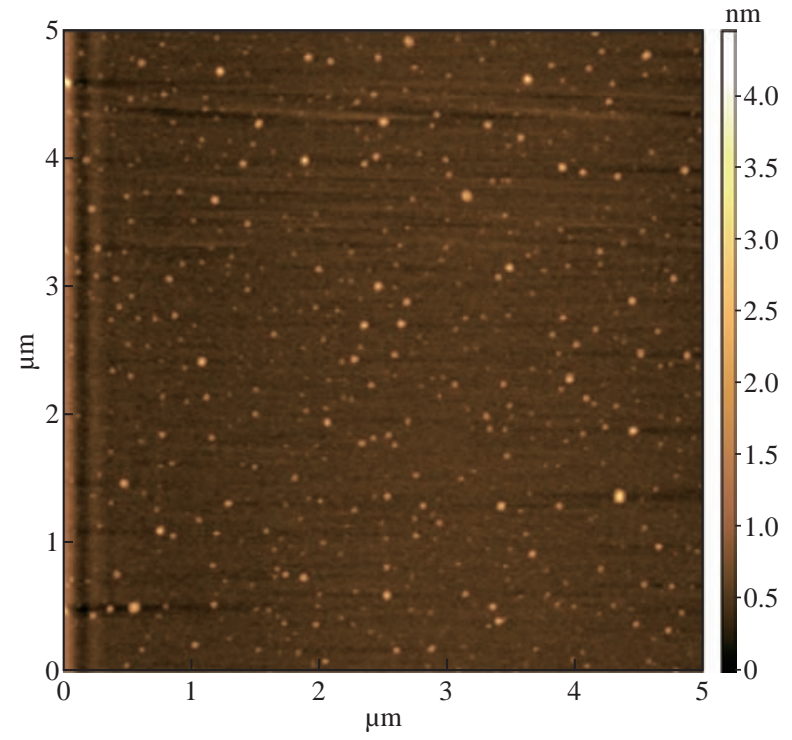

Fig. 1 Atomic force microscopy of $\mathrm{MgCl}_{2}$ nanoparticles.

rabbit blood sample in the presence of the gadolinium compound using the cyclic voltammetric technique. 2 peaks of oxidation current were observed in the cyclic voltammogram of the blood component as a result of the interaction of blood with gadolinium compound at the oxidation potential of $0.73 \mathrm{~V}$ and $0.23 \mathrm{~V}$ (Fig. 2). It can be concluded that the gadolinium compound causes oxidative stress of blood formations especially with hemoglobin in the blood; complex, which causes various symptoms for the patients such as kidney failure, stroke, or death.

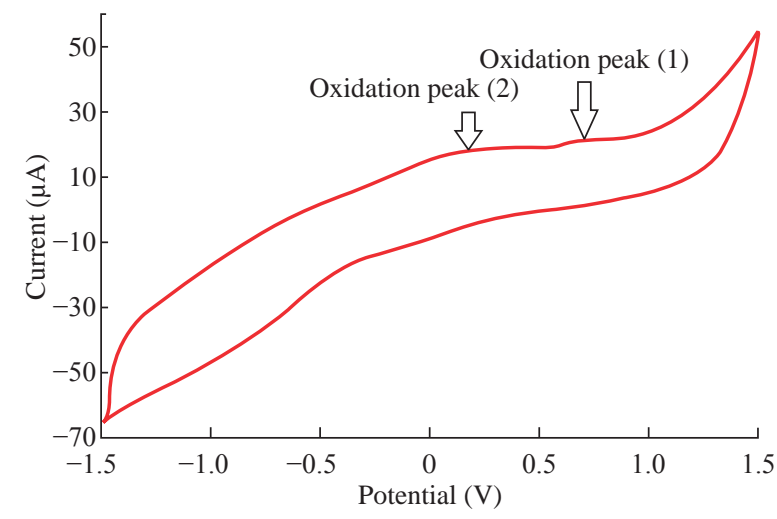

Fig. 2 Cyclic voltammogram of gadolinium compound in rabbit blood medium on glassy carbon electrode as working electrode and $\mathrm{Ag} / \mathrm{AgCl}$ as reference electrode at scan rate of $0.1 \mathrm{~V} / \mathrm{sec}$.

Gadolinium contrast medium was used to examine the MRI of the rabbit kidney, Fig. 3 and 4 illustrate the imaging of MR without and with contrast medium respectively. The gadolinium enhanced the imaging of rabbit kidney in good statues. However, gadolinium material has risk side effect yet is the only contrast used in MRI now [14].

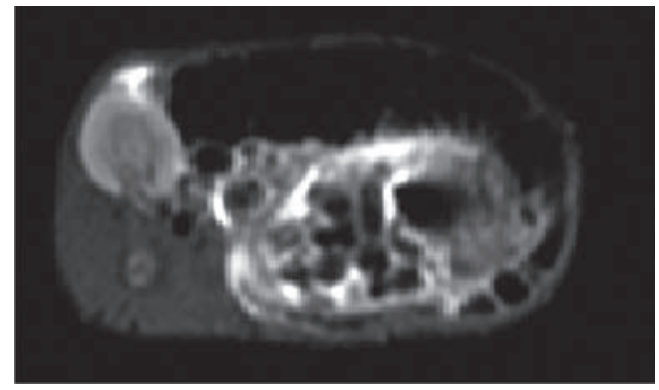

Fig. 3 Magnetic resonance imaging of rabbit kidney without contrast medium.

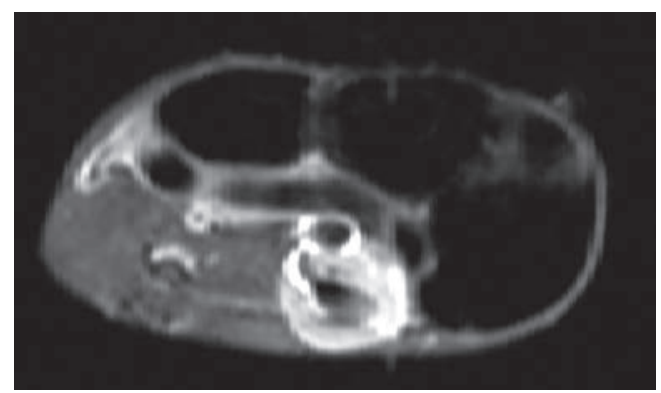

Fig. 4 Magnetic resonance imaging of rabbit kidney after injecting $2 \mathrm{~mL}$ gadolinium contrast medium.

\section{$\mathrm{MgCl}_{2}$ solution}

Magnesium chloride solution $\left(\mathrm{MgCl}_{2}\right)$ was studied as alternative contrast medium in the MRI with good chemical physical and magnetic properties [1]. The electrochemical behavior of $\mathrm{MgCl}_{2}$ in blood medium was studied by cyclic voltammetry to prove the effect of oxidation-reduction property of the compound on the component of blood samples. Fig. 5 shows the cyclic voltammogram of $\mathrm{MgCl}_{2}$ solution in blood medium. It was found that a high reduction current peak at $-0.8 \mathrm{~V}$ appeared, which means the magnesium ion in blood medium has anti-oxidative property [15]. The enhancement of MRI when using magnesium chloride as a contrast medium is illustrated in Fig. 6.

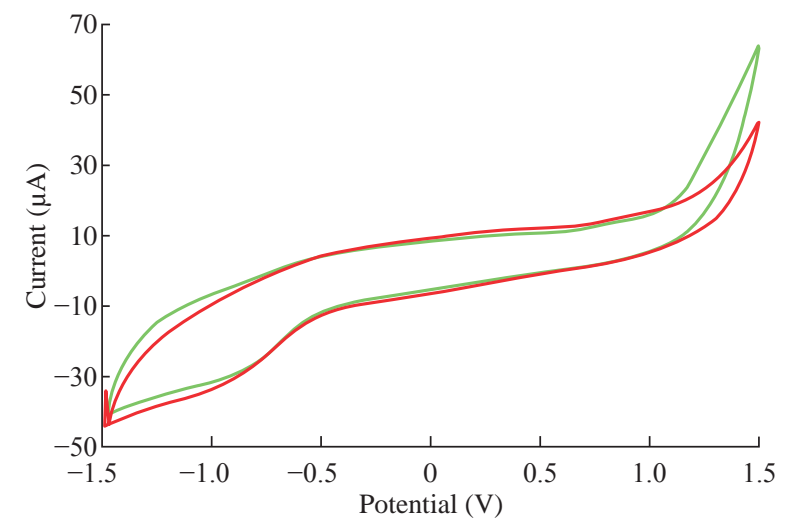

Fig. 5 Cyclic voltammogram of $\mathrm{MgCl}_{2}$ solution at different concentrations on glassy carbon electrode as working electrode and $\mathrm{Ag} / \mathrm{AgCl}$ as reference electrode at scan rate of $0.1 \mathrm{~V} / \mathrm{sec}$. 


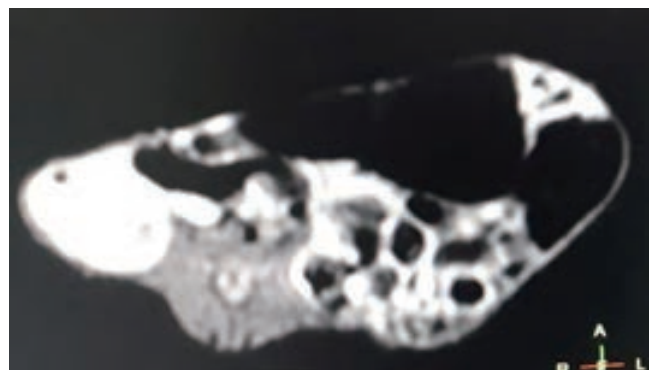

Fig. 6 Magnetic resonance imaging of kidney after injecting 2 $\mathrm{mL}$ of $\mathrm{MgCl}_{2}$ contrast medium.

In the reaction of magnesium in blood medium in the presence of hemoglobin, 2 reduction peaks appeared with iron ions of the blood component as in the following equations: [16, 17].

$\mathrm{Mg}^{0}+\mathrm{e}=\mathrm{Mg}^{1-}, \mathrm{E}=-100 \mathrm{mV}$;

$\mathrm{Mg}^{1-}+\mathrm{e}=\mathrm{Mg}^{2-}, \mathrm{E}=-250 \mathrm{mV}$.

\section{$\mathrm{MgCl}_{2}$ nanoparticles solution}

Magnesium nanoparticles can be used as an alternative contrast medium in the MRI scan to diagnose tumor and abdominal organs safely and with good visualization that helps the doctor give more brightness and high resolution to the image.

Fig. 7 illustrates the cyclic voltammogram of $\mathrm{MgCl}_{2} \mathrm{NPs}$ in normal blood medium which showed a single reduction current peak only at potential of $-1 \mathrm{~V}$; therein, magnesium nanoparticles act as anti-oxidative reagent [18].

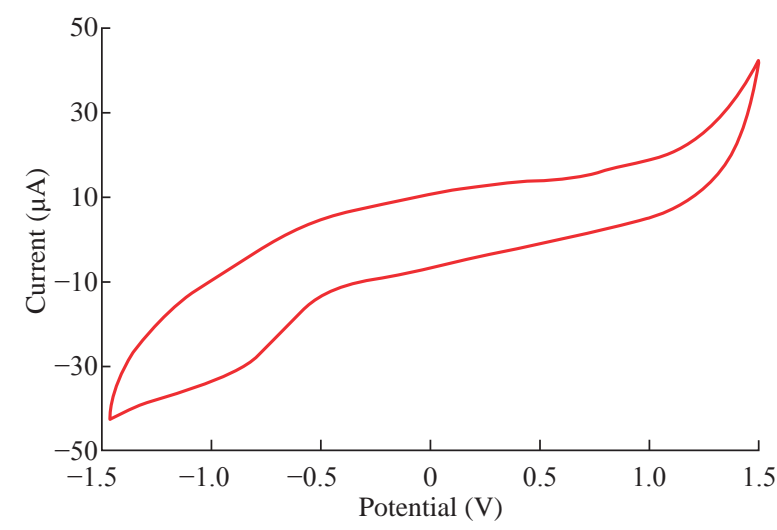

Fig. 7 Cyclic voltammogram of $\mathrm{MgCl}_{2}$ nanoparticles solution on glassy carbon electrode as working electrode and $\mathrm{Ag} / \mathrm{AgCl}$ as reference electrode at scan rate of $0.1 \mathrm{~V} / \mathrm{sec}$.

In this study, the rabbit was used in in-vivo study by MRI with nanoparticles of contrast medium $\left(\mathrm{MgCl}_{2} \mathrm{NPs}\right)$ as an alternative of gadolinium and $\mathrm{MgCl}_{2}$ solution. Imaging of MR for the rabbit kidney had a high resolution compared with gadolinium and $\mathrm{MgCl}_{2}$ solution (Fig. 8). Hence, we could use the nano contrast of $\mathrm{MgCl}_{2}$ NPs without any side effect on the health of the rabbit.

\section{Effect of ascorbic acid on $\mathrm{MgCl}_{2}$ nanoparticles}

Ascorbic acid (AA) is one of the anti-oxidant chemical compounds [19]. It can be used to trat many diseases or to avoid and save human life from the diseases [20]. In this study, AA was used to improve the role of nanoparticles in MRI. Fig. 9 shows cyclic voltammogram of $\mathrm{MgCl}_{2} \mathrm{NPs}$ with AA in blood medium which enhanced the cathodic current peak of $\mathrm{MgCl}_{2} \mathrm{NPs}$ by the anodic current peak of AA [21].

\section{Enhancement effect of $\mathrm{MgCl}_{2}$ nanoparticles on magnetic resonance imaging}

Magnesium chloride NPs were used as an alternative contrast medium in rabbits to find enhanced MRI for different sequences of kidney organ and comparison with pre- and post-use with gadolinium and $\mathrm{MgCl}_{2}$ solution. Fig. 8 and 9 show an MRI of the kidney organ of a healthy rabbit, which showed a high boost for imaging in the midst of alternative nano contrast.

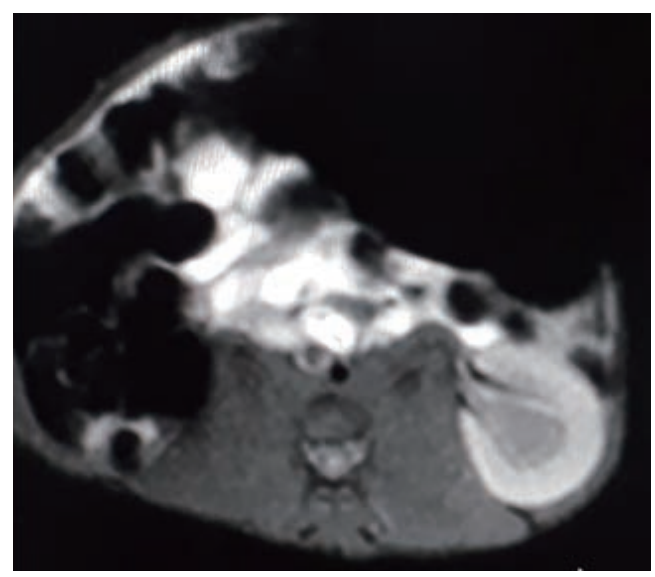

Fig. 8 Magnetic resonance imaging of kidney after injecting 2 $\mathrm{mL}$ of $\mathrm{MgCl}_{2}$ nanoparticles contrast medium (0.5 M).

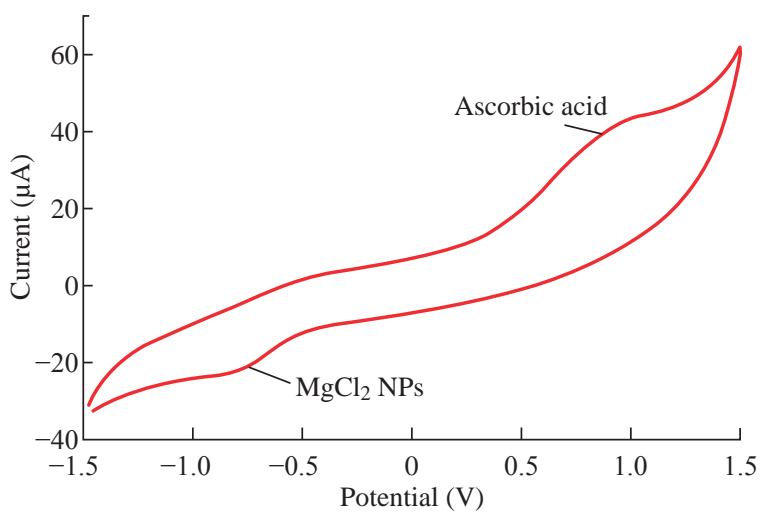

Fig. 9 Cyclic voltammogram of $\mathrm{MgCl}_{2}$ nanoparticles solution with ascorbic acid on glassy carbon electrode as working electrode and $\mathrm{Ag} / \mathrm{AgCl}$ as reference electrode at scan rate of 0.1 V/sec. 
The alternative contrast used in this research is distinguished by being free of animal side effects and giving enhanced MRI to the rabbit's kidneys compared with the other options used currently.

\section{Conclusions}

Magnesium chloride nanoparticles ( $\mathrm{MgCl}_{2} \mathrm{NPs}$ ) solution might have suitable physical and chemical properties for using as a safety contrast medium without side effects for the patients. In addition to the electrochemical properties, magnesium nanoparticles ions had a reduction current peak without oxidation peak, indicating that the alternative contrast medium when used in MRI ( $\mathrm{MgCl}_{2} \mathrm{NPs}$ ) acted as an antioxidative reagent. Also, both magnesium particles at micro- or nanoparticles enhanced the imaging for diagnosis of the abdomen organs. According to the current study, these results can be considered a major breakthrough in the field of correct diagnosis of MRI and without any effects on the patient health. It can also be concluded that it is necessary to find the appropriate alternative to the contrast media used in diagnostic imaging. We are now in the initial stage of finding the alternatives and we need more research to reach the best.

\section{References}

[1] H.B.N. Song, I.C. Hyeon, Inorganic nanoparticles for MRI contrast agents. Adv Mater, 2009, 21(21): 21332148.

[2] P.M. Kochanek, R.C. Tasker, N. Carney, et al., Guidelines for the management of pediatric severe traumatic brain injury. Neurosurgery, 2019, 1: 1169-1178.

[3] A. Semkina, A. Abakumov, N. Grinenko, et al., Magnetic resonance imaging of tumors with the use of iron oxide magnetic nanoparticles as a contrast agent. Bulletin of Experimental Biology \& Medicine, 2017, 162(6): 808811.

[4] J.P. Avila, Biomedical applications of inorganic nanoparticles: Magnetic Resonance Imaging and Hyperthermia. Ph.D thesis, University of Milano-Bicocca, 2015.

[5] H.C. Huang, S. Barua, G. Sharma, et al., Inorganic nanoparticles for cancer imaging and therapy. $J$ Control Release, 2011, 155(3): 344-357.

[6] C. Lin, S. Cai, J. Feng, Positive contrast imaging of SPIO nanoparticles. J. Nanomater, 2012, 6: 1-9.
[7] L. Zeng, D. Wu, R. Zou, et al., Paramagnetic and superparamagnetic inorganic nanoparticles for T1weighted magnetic resonance imaging. Curr Med Chem, 2018, 25(25): 2970-2986.

[8] M. Bullarbo, H. Mattson, A.K. Broman, et al., Magnesium supplementation and blood pressure in pregnancy: A double-blind randomized multicenter study. J Pregnancy, 2018: ID 4843159.

[9] M.M. Radhi, H.A.T. Al-Shimmari, E.A.J. Al-Mulla, et al., New voltammetric study of $\mathrm{MgCl}_{2}$ as alternative contrast media in MRI molecular imaging. Nano Biomed. Eng, 2017, 9(2): 152-161.

[10] H.M. Joshi, Multifunctional metal ferrite nanoparticles for MR imaging applications. J. Nanoparticle Res, 2013, 15(1): 1235.

[11] M. Botta, F. Carniato, D. Esteban-Gómez, et al., Mn (II) compounds as an alternative to Gd-based MRI probes. Future Med. Chem, 2019, 11(12): 1461-1483.

[12] H. Huang, T. Yue, Y. Xu, et al., PEG ylation of MnO nanoparticles via catechol-Mn chelation to improving T1 weighted magnetic resonance imaging application. $J$. Appl. Polym. Sci., 2015, 132(31).

[13] W. Abdelwahed, G. Degobert, S. Stainmesse et al., Freeze-drying of nanoparticles: formulation, process and storage considerations. Adva drug deliv revi., 2006, 58(15):1688-1713.

[14] H. Gustav, J.M. Willem, A.F. Geralda, et al., MRI contrast agents: current status and future perspectives. Anti-Cancer Agents Med. Chem., 2007, 7: 291-305.

[15] M.M. Radhi, E.A.J. Al-Mulla. Use of a grafted polymer electrode to study mercury ions by cyclic voltammetry. Res Chem Intermed, 2015, 41: 1413-1420,

[16] F.A. Cotton, G. Wilkinson, Advanced inorganic chemistry, 5th edition. John Wiley and Sons, 1988.

[17] A.J. Bard, L.R. Faulkner, Electrochemical methods: fundamentals and applications, 2nd edition. Wiley, 2001.

[18] Y.K. Abdul-Amir, M.M. Radhi, and E.A.J. Al-Mulla, Use of nano-sensors of the interferences between $\mathrm{Pb}((\mathrm{II})$ with each of $\mathrm{Mg}(\mathrm{II}), \mathrm{Zn}(\mathrm{II}), \mathrm{Mn}(\mathrm{II}), \mathrm{Ca}(\mathrm{II}), \mathrm{Co}(\mathrm{II})$ and PO4-3 in blood medium: An electrochemical study. Nano Biomed. Eng., 2017, 9(3): 199-207.

[19] M.M. Radhi, H.N. Abdullah, M.S. Jabir, et al., Electrochemical effect of ascorbic acid on redox current peaks of $\mathrm{CoCl}_{2}$ in blood medium. Nano Biomed. Eng., 2017, 9(2): 103-106

[20] M.M. Radhi, S.A Al-Assady, N.T. Barakat, et al., Electrochemical oxidation effect of each ascorbic acid and folic acid on lead ions in human blood. International Journal of Pharmacy and Chemistry, 2017, 3(3): 33-40.

[21] V. Sreeja, K.N. Jayaprabha, and P.A. Joy, Waterdispersible ascorbic-acid-coated magnetite nanoparticles for contrast enhancement in MRI. Appl Nanosci, 2015, 5: 435-441.

Copyright $(\subset$ Dunya Ali Mustafa, Haydar Abdulkadeer Taheer Al-Shimmari, and Muhammed Mizher Radhi. This is an openaccess article distributed under the terms of the Creative Commons Attribution License, which permits unrestricted use, distribution, and reproduction in any medium, provided the original author and source are credited. 